\title{
Gradhiva
}

GRADHI

Revue d'anthropologie et d'histoire des arts

$23 \mid 2016$

Collections mixtes

\section{In memoriam. Monsieur Fabre n'est jamais là}

\section{Giordana Charuty, Michèle Coquet et Jean Jamin}

\section{OpenEdition}

Journals

Édition électronique

URL : http://journals.openedition.org/gradhiva/3123

DOI : 10.4000/gradhiva.3123

ISSN : 1760-849X

\section{Éditeur}

Musée du quai Branly Jacques Chirac

\section{Édition imprimée}

Date de publication : 25 mai 2016

Pagination : $2-25$

ISBN : 978-2-35744-093-7

ISSN : 0764-8928

Référence électronique

Giordana Charuty, Michèle Coquet et Jean Jamin, «In memoriam. Monsieur Fabre n'est jamais là », Gradhiva [En ligne], 23 | 2016, mis en ligne le 25 mai 2016, consulté le 24 septembre 2020. URL : http:// journals.openedition.org/gradhiva/3123; DOI : https://doi.org/10.4000/gradhiva.3123 


\section{In memoriam. Monsieur Fabre n'est jamais là}

par Giordana Charuty, Michèle Coquet et Jean Jamin

Le musée qui autrefois me faisait songer au bordel, serait plutôt pour moi - aujourd'hui - une église.

Solution ancienne: se dépenser;

solution actuelle: se recueillir.

Michel Leiris, Journal 1922-1989, Paris, Gallimard, 1992

\section{Envoi}

Le titre de cet hommage à notre ami et collègue Daniel Fabre est une allusion à l'écriteau que Blaise Cendrars, dans les années 1920, aurait fait apposer sur la porte de son bureau aux Éditions de la Sirène où devaient figurer, comme sur celle de ses collaborateurs et complices, notamment Félix Fénéon, leurs jours et heures de réception. Au lieu de cela, il aurait été inscrit: «Monsieur Cendrars n'est jamais là. » Cette anecdote est rapportée par l'un des jeunes admirateurs belges du poète, Robert Guiette, dans un livre de souvenirs sur lui et leurs déambulations nocturnes en compagnie de Fernand Léger dans les rues, bistrots et bastringues parisiens, et qui reprend cette formule pour titre $\mathbf{1}^{\mathbf{f i g} .2)}$.

Retrouvé chez un bouquiniste, ce livre avait été offert par Daniel Fabre - qui aimait beaucoup Cendrars, tant le «bourlingueur» que le poète, chroniqueur et romancier - à l'un des auteurs de cet hommage. Lui-même se reprochait d'ailleurs, non sans quelque malice de brigandeau (telle celle de son bonhomme de Cavanac $^{2}$ ) et cet humour dadaïste qui faisaient partie des traits saillants de sa personnalité, de ne pas avoir eu l'idée de mettre un panneau de cet acabit sur la porte des différents bureaux qu'il avait théoriquement occupés, mais dont il eut l'art et la manière de continûment s'absenter, comme s'il fallait abolir la loi qui interdisait d'« occuper plusieurs lieux à la fois ", ou d'être là sans jamais l'être vraiment... Ce que, paradoxalement, avait préconisé le «reclus» de Carcassonne, le poète et écrivain Joë Bousquet, dont l'existence et «l'espace arachnéen » dans lequel elle se déploya et que Daniel Fabre se plaisait à imaginer et même à décrire jouèrent un rôle important dans la formation et la sensibilité littéraire du jeune Daniel, à travers notamment l'œuvre et l'enseignement de René Nelli, qui fut très proche de Bousquet ${ }^{3}$.

Aussi ne pouvons-nous nous résoudre à le voir partir sans lui offrir, en guise de contre-don, cet écriteau qu'il ne trouva jamais le temps d'afficher mais qui lui colle tellement à la peau et qu'on regrette de ne pas avoir su en deviner toute l'ironie ni en comprendre toutes les implications au moment où il nous en parlait, se frottant les mains entre le tiramisu et la «noisette", avec son rire flûté aux inflexions languedociennes qui invitait à ce qu'on se précipitât pour en clouer une réplique sur la porte de son bureau.
1. Monsieur Cendrars n'est jamais là, Montpellier, Éditions du Limon, [1922] 1990.

2. Voir Dominique Blanc et Daniel Fabre (éd.), Le Brigand de Cavanac. Le fait divers, le roman, l'histoire, Lagrasse, Verdier, 2015 [1982].

3. Voir infra et Daniel Fabre, "D'une jeunesse étonnante", présentation de la réimpression intégrale de la revue Chantiers (1928-1930), Paris, Jean-Michel Place, 1987 : IX-L. Voir également Daniel Fabre et Jean-Pierre Piniès (éd.), René Nelli et les Cahiers du Sud, Carcassonne, Garae Hésiode, 1987. 
4. Le nombre des articles publiés par Daniel Fabre est impressionnant (près de deux cents), la plupart dans des revues dites "à comité de lecture"; leur diversité l'est tout autant. Diversité et non pas disparité ni hétérogénéité: tous sont portés par un même style, traversés par une même méthode, habités par une même passion, organisés par une même pensée,

comme si - infatigable scripteur et lecteur - Fabre voulait à leur propos se mesurer avec la quadrature du cercle: les tirer à la fois vers l'argumentation et la narration - d'eux faire des manières de contes.
5. Né à Narbonne le 21 février 1947, il est mort à Toulouse le 23 janvier 2016. II allait avoir 69 ans et, en septembre de cette année, devait s'apprêter à partir à la retraite, sans vraiment le croire ni le vouloir, ni même l'imaginer. Pas plus qu'il ne fut là dans ses différents bureaux, il ne pouvait y avoir de "retraite" où il aurait été là.

6. Mot qu'affectionnait particulièrement Daniel Fabre, au point de l'utiliser dans le titre d'un des ouvrages collectifs qu'il a dirigé avec Pierre Centlivres et Françoise Zonabend (La Fabrique des héros, Paris, Maison des sciences de l'homme, 1998, voir infra). Lors d'un court hommage rendu à Daniel Fabre sur France Culture, le lundi 25 janvier - le surlendemain de sa mort -, le journaliste et producteur Emmanuel Laurentin reconnaissait que c'est à lui qu'il avait pensé en intitulant son émission "La fabrique de l'histoire".

\section{ci-contre}

fig. 1

Daniel Fabre en novembre 2011. D.R.
À l'image de Cendrars, Daniel Fabre était un buissonnier de la vie comme il était un dandy de la pensée et de l'écriture. Nul autre, mieux que lui, n'était capable d'aller des écrits de Montesquieu à ceux de John Millington Synge en passant par des barbouilleux d'Europe centrale ou par d'obscurs auteurs de récits autobiographiques des Pouilles ou d'ailleurs, sans s'écarter de l'égard que l'on doit à toutes choses humaines ni - que celles-ci soient grandes ou petites - de la rigueur qu'exige leur analyse ${ }^{4}$. Dandy certes, il en avait le goût de l'intervalle et de l'extravagance, mais nullement le penchant de l'arrogance.

\section{La fabrique}

Ethnographe et anthropologue, directeur d'études à l'École des hautes études en sciences sociales où il occupait une chaire d'ethnologie de l'Europe, Daniel Fabre ${ }^{5}$ a incarné une alliance rare de plusieurs qualités: une très grande liberté d'écrire, de dire et de penser associée à une infatigable énergie mise, avec générosité, au service de grandes entreprises collectives que ravivait constamment une singulière capacité d'innovation non seulement en débusquant des objets ordinaires, au statut épistémologique faible sinon trivial, et qui, sous sa plume ou dans son verbe, prenaient alors de belles lettres de noblesse (écritures ordinaires, chambres, tables et fioles d'écrivains, aquarelles, gouaches de grenier ou croûtes de salle à manger, brigandages, faits divers, carnavals, chansons populaires, etc.), mais en n'hésitant pas à faire bouger les lignes disciplinaires, bondissant de l'ethnologie la plus classique à l'histoire la plus mouvante, sans négliger la littérature, la philologie, la linguistique, l'histoire de l'art et même l'ethnomusicologie ou la préhistoire - le tout avec une exceptionnelle maîtrise de la langue et de la culture, en un mot: des humanités, ouvert à l'entière gamme des vibrations du monde, de l'histoire et des sociétés.

Daniel Fabre était de ces savants joyeux et érudits - joyeux parce qu'érudits (un "gai savoir") - qui, dans la tradition des Jacques Le Goff, Jean-Pierre Vernant, Marc Augé, Roger Chartier ou Emmanuel Terray, pour ne citer qu'eux, se lançaient dans l'oral comme dans l'écrit ainsi qu'un fournier prépare, pétrit et veille à la température de sa pâte: la cuire et la livrer à point, comme si tout ceci - le travail d'écriture, le fil de l'enquête, les tâtonnements de la recherche, les embarras de la pensée - relevait de la fabrique plutôt que de l'académie. La fabrique ${ }^{6}$, l'atelier, l'ouvroir que l'on partage dans la fournaise ou dans les frimas mais toujours dans la proximité des pensées, des dits et des écrits. Avec lui, auprès de lui, on se sentait tous un peu mitrons.

Plusieurs générations de chercheurs et de doctorants, dans une équipe de laboratoire, un comité éditorial, un conseil scientifique, une mission ministérielle, ont donc fait l'expérience gratifiante qu'entrer dans une relation de travail avec Daniel Fabre, c'était voir gaiement s'écrouler les discours trop ressassés pour, de ces gravats, faire surgir des mondes tout neufs. Chantiers improbables dont rien ne garantissait qu'ils atteindraient chapiteaux et faîtages, mais qui animaient quiconque s'y ralliait d'une même ardeur. Quel en était le secret? Une "conversion du regard", des «rencontres fécondes", aimait-il dire. Un immense bagage doublé d'un subtil savoir-faire et vivre, doit-on ajouter, pour relier des lieux, des temps, 


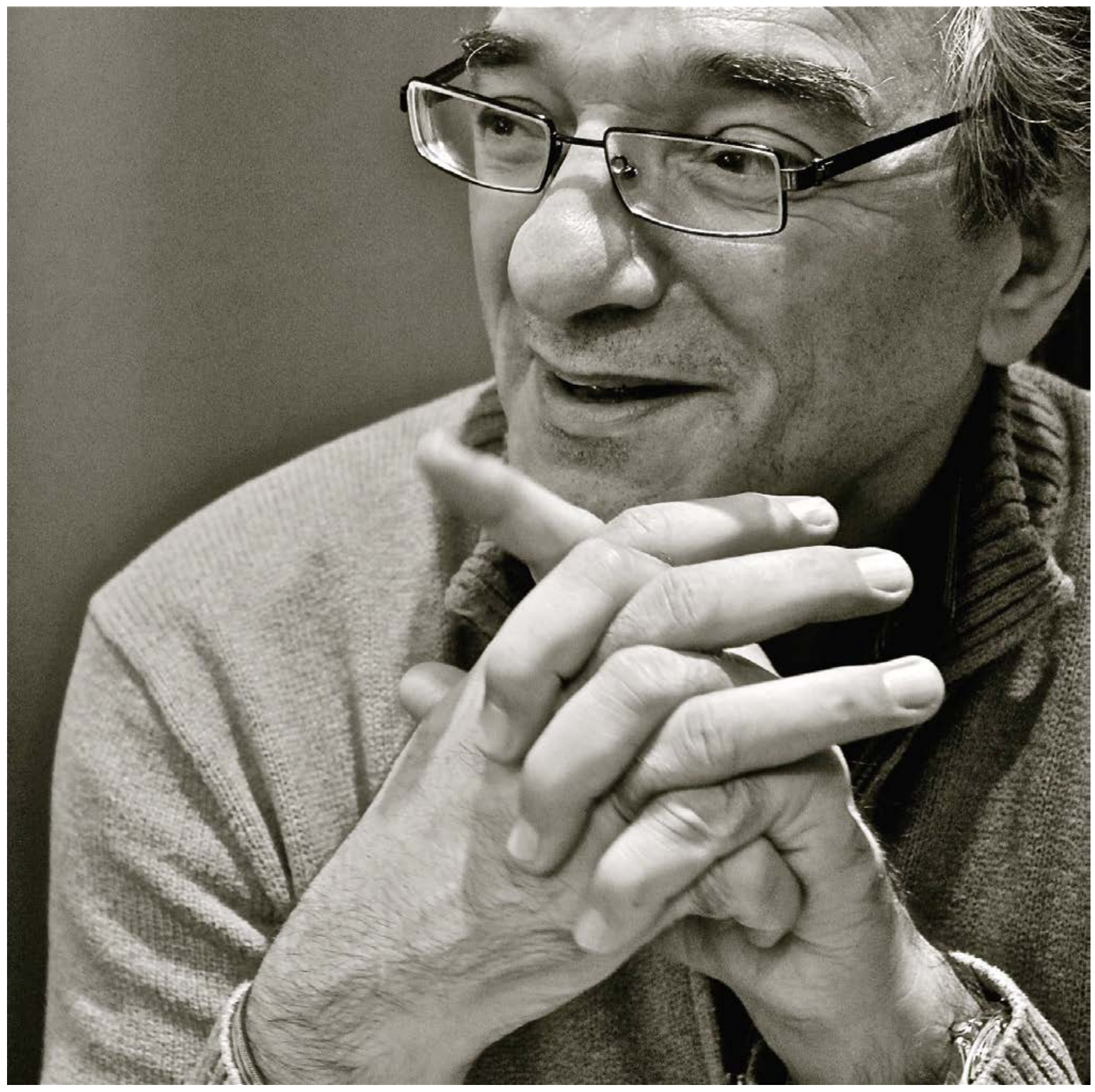




\section{Robert Guiette}

\section{«Monsieur Cendrars \\ n'est jamais là "}

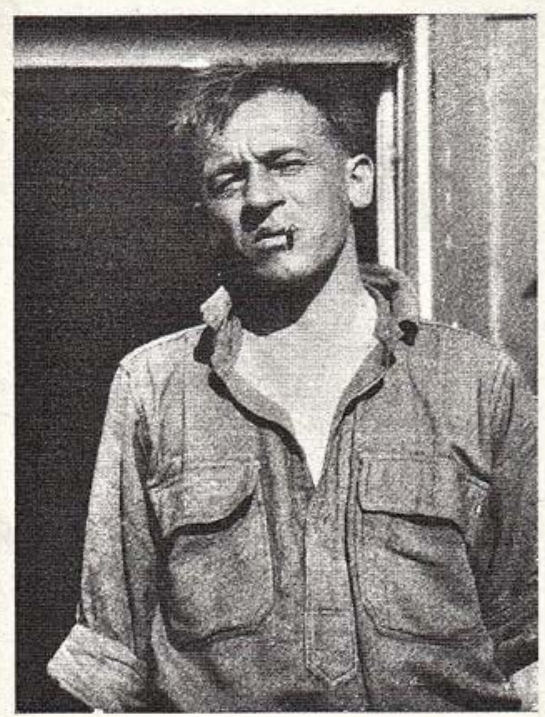

fig. 2

Couverture du livre de Robert Guiette, "Monsieur Cendrars n'est jamais là", texte établi et présenté par Michel Décaudin. Montpellier, éditions du Limon, 1990.

\section{éditions du limon}


des personnes aux pensées et tempéraments parfois contrastés, des savoirs hérissés de frontières, balisés de chemins creux comme ceux qu'avec Claudine Fabre-Vassas il avait suivis et franchis à travers l'œuvre posthume d'Yvonne Verdier, elle-même plongée dans l'analyse des romans de Thomas Hardy et de son Wessex imaginaire ${ }^{7}$, où affleurait une interrogation fondamentale sur le passage d'une société dans laquelle la coutume et le destin formaient un cercle concentrique qui écrasait la vie et, parfois, la mutilait tragiquement, à «une société des individus » où chacun pouvait prétendre «devenir soi-même» un jour - creuset de la liberté et de la modernité au risque de la rupture, comme cela arrive aux héros et héroïnes de Thomas Hardy. Au fond, qu'est-ce qui se passe si l'on s'écarte de la coutume et des rites?

C'est une des questions qu'inlassablement Daniel Fabre reprendra à travers son ethnographie et ses études des communautés rurales ou des traditions orales, peut-être wittgensteinien malgré lui, en démontrant que ce n'est pas la tradition qui dit ce qu'il faut faire précisément pour suivre la tradition... Et c'est là un des points de départ pour toute une anthropologie de la littérature qu'il appelait de ses vœux, et en particulier des romans dont les univers forcément clos qu'ils décrivent (jamais personne n'empêchera le Pequod de Moby Dick de sombrer, ni Fantine de vendre ses dents et ses cheveux pour payer aux Thénardier la pension de Cosette) ne cessent, au fil du temps, d'ouvrir des fenêtres sur le nôtre, hic et nunc. Cela, l'anthropologie ne pouvait le manquer. Lui, en tout cas, ne manqua pas, comme parallèlement le fit Umberto Eco en sémiologue et linguiste, de s'interroger en ethnologue sur la manière dont des œuvres de fiction (histoires, paysages, personnages) se voient dotées de «permis de séjour» dans notre réalité et viennent s'y lover durablement ${ }^{\mathbf{8}}$, sans omettre non plus le processus en quelque sorte inverse, c'est-à-dire, pour reprendre une expression de Truman Capote et comprendre sa propre démarche dans le traitement de faits divers, comment peuvent s'écrire des «romans vrais» ou des «romans documentaires " (non-fiction novels ${ }^{9}$ ).

\section{Une ethnologie de soi}

Comment devient-on l'ethnologue de sa propre culture? Très tôt formulée, la question nourrissait chez Daniel Fabre une réflexion épistémologique dont l'exigence ne l'a jamais quitté. Elle s'est, certes, complexifiée dans le temps mais une réponse fait retour, comme un leitmotiv. Jeune enseignant de lettres et d'histoire, Daniel Fabre part, au milieu des années 1960,dans la quête improbable des récits occitans de la tradition orale européenne. Défaisant tous les diagnostics sur leur disparition, le pacte ethnographique qu'il sut instaurer rendit la mémoire à des narrateurs, hommes et femmes, au répertoire et aux compétences bien individualisés. Au grand dam de Claude Brémond ${ }^{\mathbf{1 0}}$, qui percevait un réquisitoire déplacé dans une recherche maîtrisant, par ailleurs, toutes les techniques philologiques, l'enquête tournait le dos au modèle naturaliste de la collecte, pour qualifier d'« ethnocidaire» - terme qui venait d'être introduit par Robert Jaulin ${ }^{\mathbf{1 1}}$ - la disparition des anciennes sociétés agropastorales de montagne ${ }^{\mathbf{1 2}}$. Plus récemment, le retenait la convergence entre la posture de ces derniers conteurs inscrits dans leur société et un modèle impensé de l'anthropologie - le paradigme des derniers - qu'il s'employait à mettre en évidence.
7. Voir Yvonne Verdier, Coutume et destin. Thomas Hardy et autres essais, Paris, Gallimard, 1995, précédé de Claudine Fabre-Vassas et Daniel Fabre, «Du rite au roman " : 7-37.

8. Voir Daniel Fabre,

"Peindre la mémoire". L'Homme 175-176, 2005: 251-276; Daniel Fabre, Marcello Massenzio et Jean-Claude Schmitt, «Autobiographie, histoire et fiction. Entretien ", L'Homme 195-196: 83-102.

De là également son intérêt pour les «transferts de sacralité" (de l'univers religieux au monde laïque et notamment à l'univers tangible des fabricants d'œuvres de l'esprit, peintres, écrivains, poètes) dont il fit un thème de recherche dominant dans ses séminaires de l'École des hautes études en sciences sociales au début des années 2000 (voir Daniel Fabre, "Maisons d'écrivains", Le Débat 115, 2001: 172-177).

9. Voir Truman Capote, De sang-froid. Récit véridique d'un meurtre multiple et de ses conséquences, Paris, Gallimard, 1965.

10. Voir Claude Brémond Logique du récit, Paris, Seuil, 1973.

11. Voir Robert Jaulin, La Paix blanche, Introduction à l'ethnocide, Paris, Seuil, 1970.

12. Voir Daniel Fabre et Jacques Lacroix, La Tradition orale du conte occitan. Les Pyrénées audoises, Paris, PUFPublications de l'Institut d'études occitanes, 1975. Compte rendu de Claude Brémond, Annales ESC, 1977, vol. 32, n³: 555-556. 
fig. 3

Fernand Léger, La Lecture, 1924, huile sur toile, $113.5 \times 146 \mathrm{~cm}$. Paris, musée national d'Art moderne-Centre de création industrielle. Photo () Centre Pompidou, MNAM-CCI, Dist. RMN-Grand Palais

Jacques Faujour () ADAGP, Paris, 2016

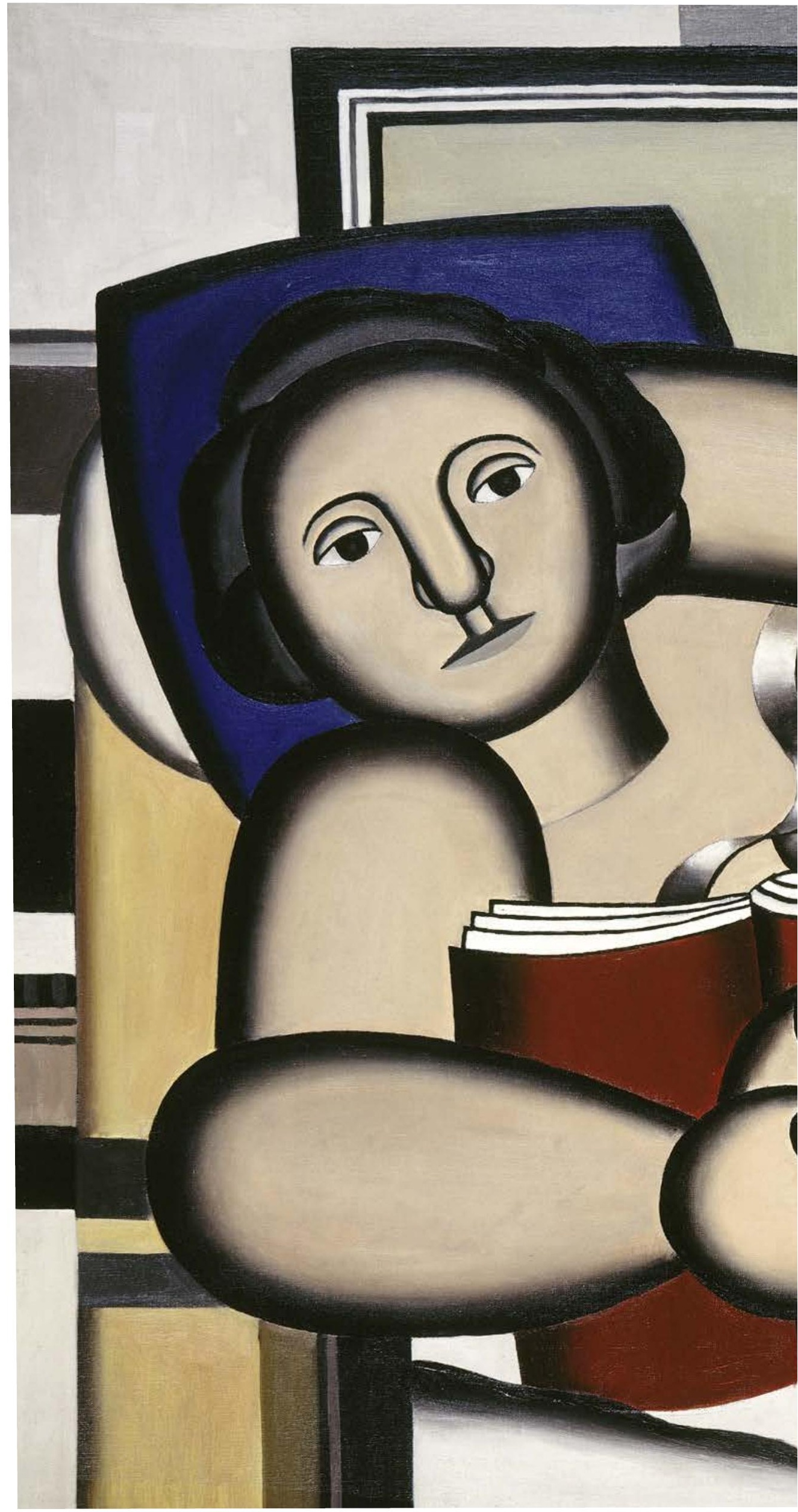




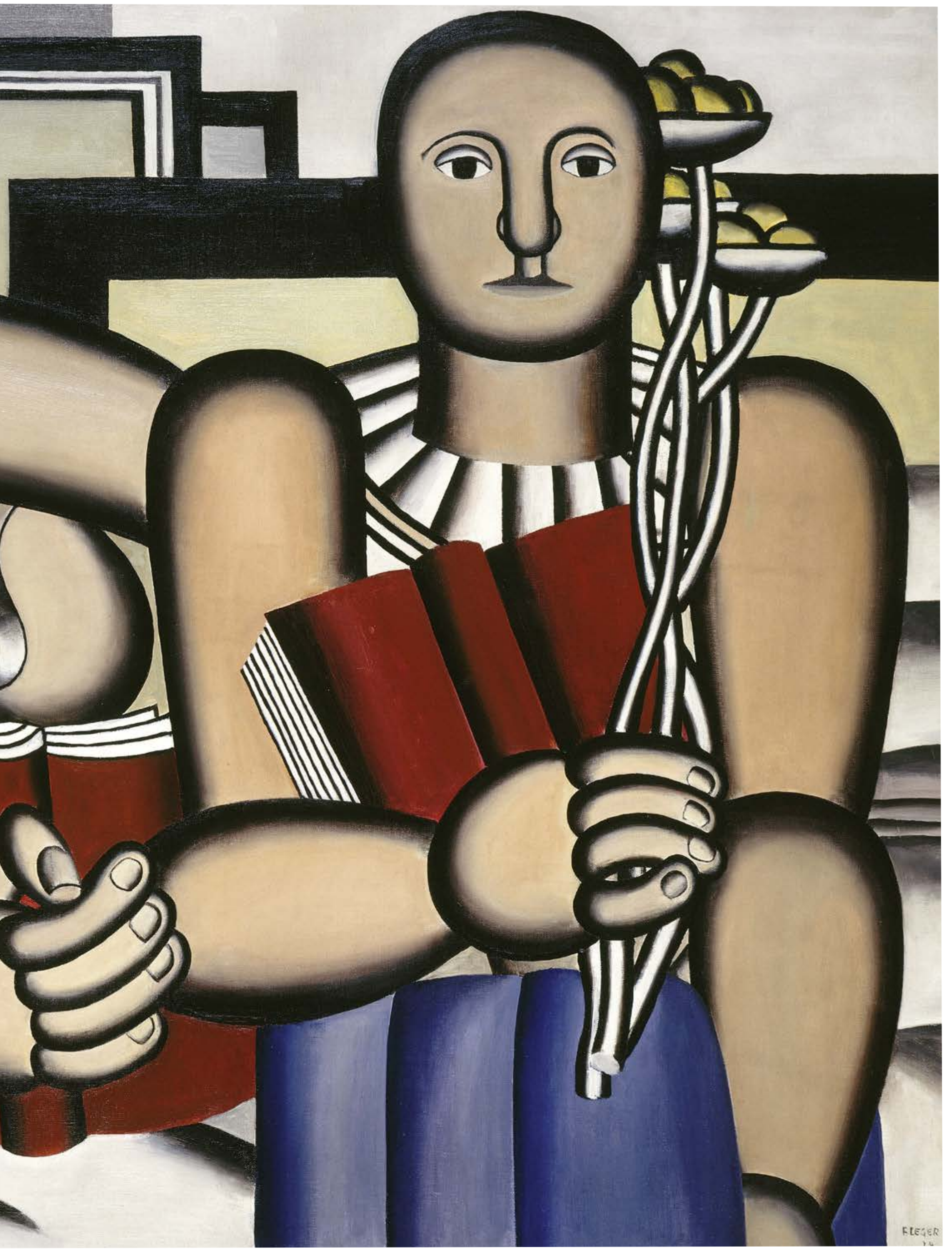




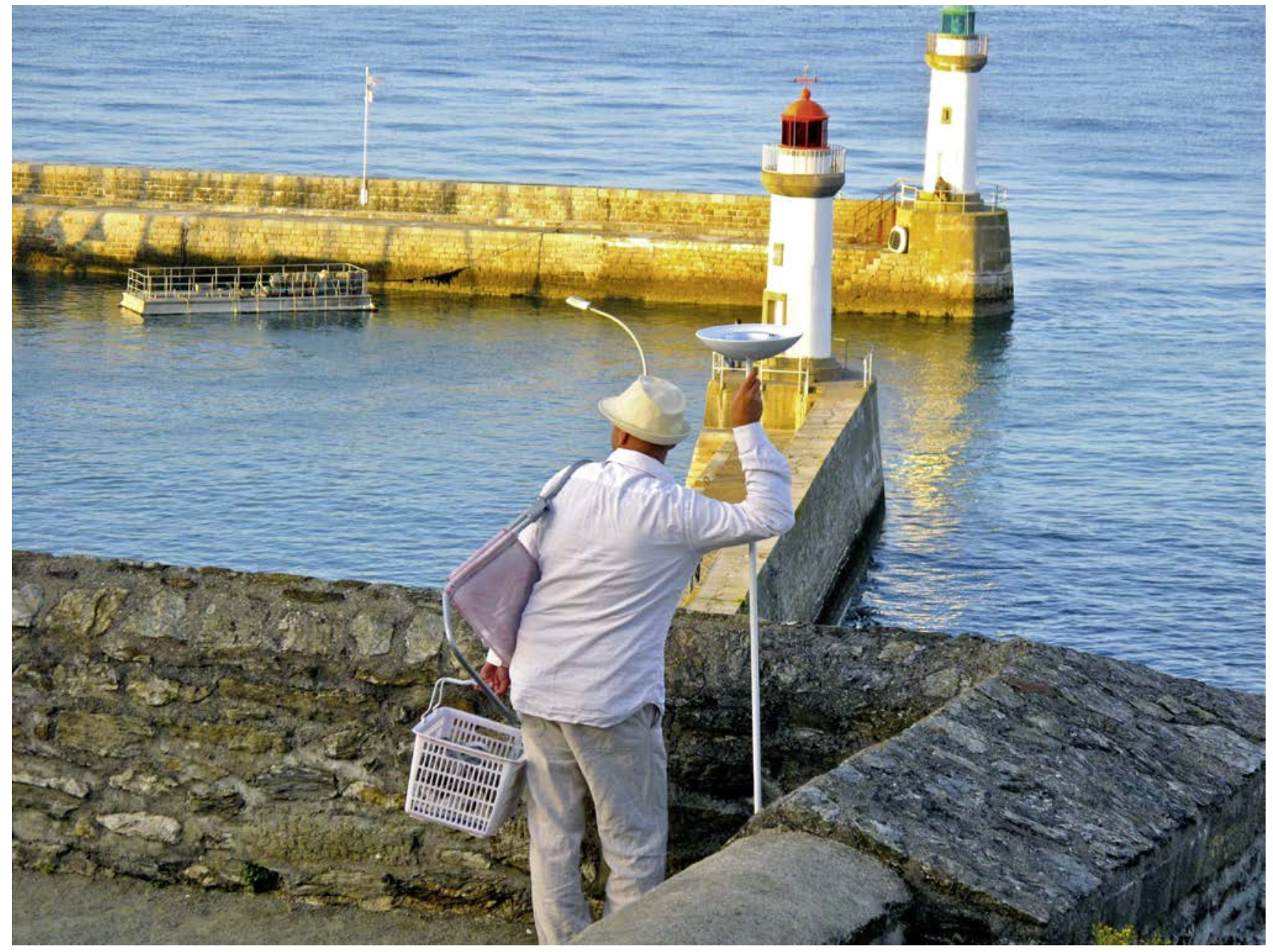


À la fin des années 1970, après de multiples enquêtes sur le renouveau de la fête languedocienne, le jeune ethnologue de 30 ans faisait le constat étonné que, du carnaval narbonnais de ses 8 ans où il figurait en Chinois au «tour de l'âne" de la cité carcassonnaise, réservé aux mariés de l'année, il n'avait jamais abandonné la «fête vécue ». Constat qu'il transformait, immédiatement, en une proposition générale: «Sans doute toute recherche ne peut que désirer, parfois au terme d'obscurs détours, répéter quelques passages d'une vie pour tenter de les inscrire en soi, de les comprendre ${ }^{13}$." Vingt ans plus tard, participant avec Jean-Claude Schmitt à un débat autour de L'Histoire des jeunes en Occident dans le cadre des "Lundis de l'histoire", l'émission animée par Jacques Le Goff sur France Culture, l'anthropologue révélait à ses amis médiévistes que les propositions générales qu'il formulait à partir de l'observation de la sociabilité juvénile dans un village de la montagne Noire des années 1960 reposaient sur une minutieuse auto-ethnographie ${ }^{14}$. Ce qu'il ne manqua pas, tout récemment, de rappeler pour le public des conférences du campus Condorcet ${ }^{15}$.

Outre ces aveux, on pourrait multiplier les relevés de traces autobiographiques dans la constellation des expériences que Daniel Fabre a systématiquement identifiées et documentées dans leur double dimension de trajectoire individuelle - des manières de garçons - et de charge collective d'un groupe social, la jeunesse - assurer la cohésion communautaire -, en inscrivant leurs réalisations languedociennes, rurales ou urbaines, dans de plus amples architectures symboliques. Mais ce vécu a, d'abord, pris sens dans une conjoncture politique bien précise: la dimension contestataire,tout au long des années 1970, d'une interrogation militante sur les «minorités culturelles» au sein des États-nations. En Languedoc, la fièvre créatrice de ces mouvements trouvait chez l'enseignant de lettres, qui s'était spécialisé en études romanes avant de devenir l'ethnographe de la fête, des littératures orales et des justices coutumières, un généreux interlocuteur dispensant sans compter aux amis écrivains, acteurs de théâtre, chanteurs d'une renaissance occitane les tout derniers résultats de ses recherches de terrain, de bibliothèques ou d'archives qu'il fréquentait tout aussi assidûment.

Comment ce moment de libération des langues et des corps auraitil pu laisser indifférent un jeune intellectuel rebelle, familiarisé dans son enfance narbonnaise avec les parlers catalan, occitan, italien dont résonnait la maison de la rue des Bons-Enfants? Aussi bien se faisait-il, au sein des instances nationales de la recherche, l'ardent défenseur d'une pratique ethnographique qui, en France, ne soit plus aveugle au pluralisme linguistique ni aux situations de diglossie, en somme une ethnographie attentive à la langue des émotions incarnées ${ }^{16}$.

Mais, de manière plus secrète ou plus intime, la voie de l'ethnologie a aussi emprunté, pour Daniel Fabre, ces affinités avec les avant-gardes littéraires et artistiques que l'on interroge plutôt à partir de l'histoire de l'africanisme ou de la musique de jazz ${ }^{\mathbf{1 7}}$ et qui, s'agissant de l'ethnologie de l'Europe, attendent encore leur analyste. Ainsi, le 31 décembre 1978, la chambre du "gisant de la rue de Verdun", l'écrivain et poète Joë Bousquet, restée close depuis sa mort en 1950, s'ouvrait à l'ethnologue invité à fêter
13. Voir Daniel Fabre et Charles Camberoque, La Fête en Languedoc. Regards sur le carnaval aujourd'hui, Toulouse, Privat, 1977: 10.

14. Voir Daniel Fabre, " "Faire la jeunesse" au village ", in Giovanni Levi et Jean-Claude Schmitt (éd.), Histoire des jeunes en Occident, Paris, Seuil, 1996, t. II : 51-83. Voir également Daniel Fabre, «Fondu au noir ", L'Homme 191, 2009: 27-36, et id.,

"Rock des villes, rock des champs ", L'Homme 215-216: 233-250.

\section{Voir infra.}

16. Voir Daniel Fabre, "Les minorités nationales en pays industrialisés ", in L'Anthropologie en France. Situation actuelle et avenir. Paris 18-22 avril 1977, Paris, Éditions du CNRS ("Colloques internationaux du CNRS 573»), 1979: 293-314.

17. Voir Jean Jamin "L'Afrique en tête", L'Homme 185-186, 2008: 401-440.

\section{page 2 et ci-contre}

fig. 4

L'Allumeur de réverbères (cl. Jean Jamin, Belle-Île, août 2014). «Peut-être bien que cet homme est absurde. Cependant il est moins absurde que le roi, que le vaniteux que le businessman et que le buveur.

Au moins son travail a-t-il un sens. Quand il allume son réverbère, c'est comme s'il faisait naître une étoile de plus, ou une fleur. Quand il éteint son réverbère ça endort la fleur ou l'étoile. C'est une occupation très jolie. C'est véritablement utile puisque c'est joli. » Antoine de Saint-Exupéry, Le Petit Prince, Paris, Gallimard, 1946 
18. Daniel Fabre a fait le récit de cette visite posthume: "Face au double", Vies et revies de René Nelli, Carcassonne, Garae Hésiode, 2011: 69-74.

19. Voir Joë Bousquet présenté par Michel Maurette. Lettres inédites. Une bibliographie. Visages de ce temps, Rodez, Éditions

Subervie, 1963; René Nelli, L'Érotique des troubadours, Toulouse, Privat, 1963.

20. La réimpression intégrale de la revue, sous la direction de Daniel Fabre et avec une présentation substantielle de lui, a été réalisée aux Éditions Jean-Michel Place en 1987. Voir supra, note 3.

21. Voir Daniel Fabre, "Accents de Jean Guilaine ", in Daniel Fabre (éd.), De Méditerranée et d'ailleurs... Mélanges offerts à Jean Guilaine, Toulouse, Archives d'écologie préhistorique, 2009: 1-10.

22. Dont le numéro d'Études rurales (9798, 1985) consacré au texte ethnographique (Jean Jamin et Françoise Zonabend, éd.) fut en quelque sorte à la fois les actes et le prolongement.

\section{ci-contre}

fig. 5

L'affiche du film d'animation sorti en 1980. D.R. la nouvelle année chez la sœur de l'écrivain, avec quelques amis proches. À leur tête, le poète et philosophe René Nelli qui en incarnait, entre Carcassonne et Toulouse, la présence toujours active, comme Daniel Fabre le rappelait récemment: "Je voyais surtout dans Nelli, au début de nos relations régulières, celui par qui Bousquet revivait, exactement comme s'incarne un mythe $\mathbf{1 8}^{\mathbf{1 8}}$ » Ce mythe avait pris corps à la fin de l'adolescence avec le témoignage de l'écrivain-paysan Michel Maurette, désireux de transmettre au lecteur «l'enchantement de la chambre» et, à travers René Nelli, il se trouvait associé à la lecture bouleversante de L'Érotique des troubadours ${ }^{19}$.

Cultiver, sur le mode distancié, cette double fascination, c'était faire sienne la singulière conjonction entre le poétique et l'ethnographique dont Carcassonne fut, entre les deux guerres, l'improbable lieu de naissance. Côté poétique, portée par le surréalisme, c'est la création autour de Bousquet de Chantiers 20 (1928-1930), brève revue d'une "jeunesse étonnante", disait Paul Éluard, qui se prolongera, avec René Nelli, dans les Cahiers du Sud de Jean Ballard. Côté ethnographique, portée par le Front populaire, c'est la création de la revue Folklore (1938), dont René Nelli fut durant de longues années le secrétaire général, puis, après la guerre, celle de l'Institut d'études occitanes que présida Jean Cassou entre 1945 et 1952. Penseur de la dissidence cathare, de l'amour courtois et de la poétique des troubadours, René Nelli dispensa à l'université de Toulouse, entre 1945 et 1974, un cours d'ethnographie méridionale qui se prolongeait dans un bistrot de la place du Capitole. Quelques années plus tard, au même jour et dans les mêmes lieux, l'enseignement de Daniel Fabre en prit la relève, la polygraphie, la quête spirituelle et le dilettantisme en moins. Le cadre en était, cette fois, l'École des hautes études en sciences sociales et le Centre national de la recherche scientifique, par le biais des diverses créations institutionnelles du protohistorien Jean Guilaine, le voisin de Ladern (Aude), qui avait luimême beaucoup fréquenté Nelli dans les années 1960, et qui ne se départira jamais, même au Collège de France, des traces de ses langues bariolées de l'enfance 21 .

«Petits» et "grands» séminaires du jeudi après-midi, entrecoupés et suivis d'échanges fiévreux sur l'avancée du travail durant la semaine écoulée, les lectures, les rencontres et les mises à l'épreuve dans les séminaires parisiens devinrent à leur tour, tout au long des années 1980, autant de rendez-vous qu'aucun membre de l'équipe, pas plus qu'aucun chercheur invité, n'aurait manqués. Bon nombre de mémorables programmes d'enquête - "Le roi des oiseaux", "Les primitifs de l'ethnologie", "Le retour des morts", "L'illettré savant» - reformulaient et transformaient de fond en comble, à l'insu de la plupart des auditeurs, des thèmes de prédilection de celui qui, aux côtés de Joë Bousquet, avait durant la guerre entretenu l'esprit de résistance. Et c'était bien à une semblable indiscipline, à une même insoumission, que conviait l'organisateur du premier grand colloque de Toulouse en sciences humaines en 1982: «Voies nouvelles en ethnologie de la France ${ }^{22}$ ».

Un peu plus tard encore, réalisant l'ubiquité rêvée par Joë Bousquet, Daniel Fabre installera l'un de ses bureaux exactement au-dessus de la chambre du héros de la littérature moderne. II pouvait, désormais, se faire 


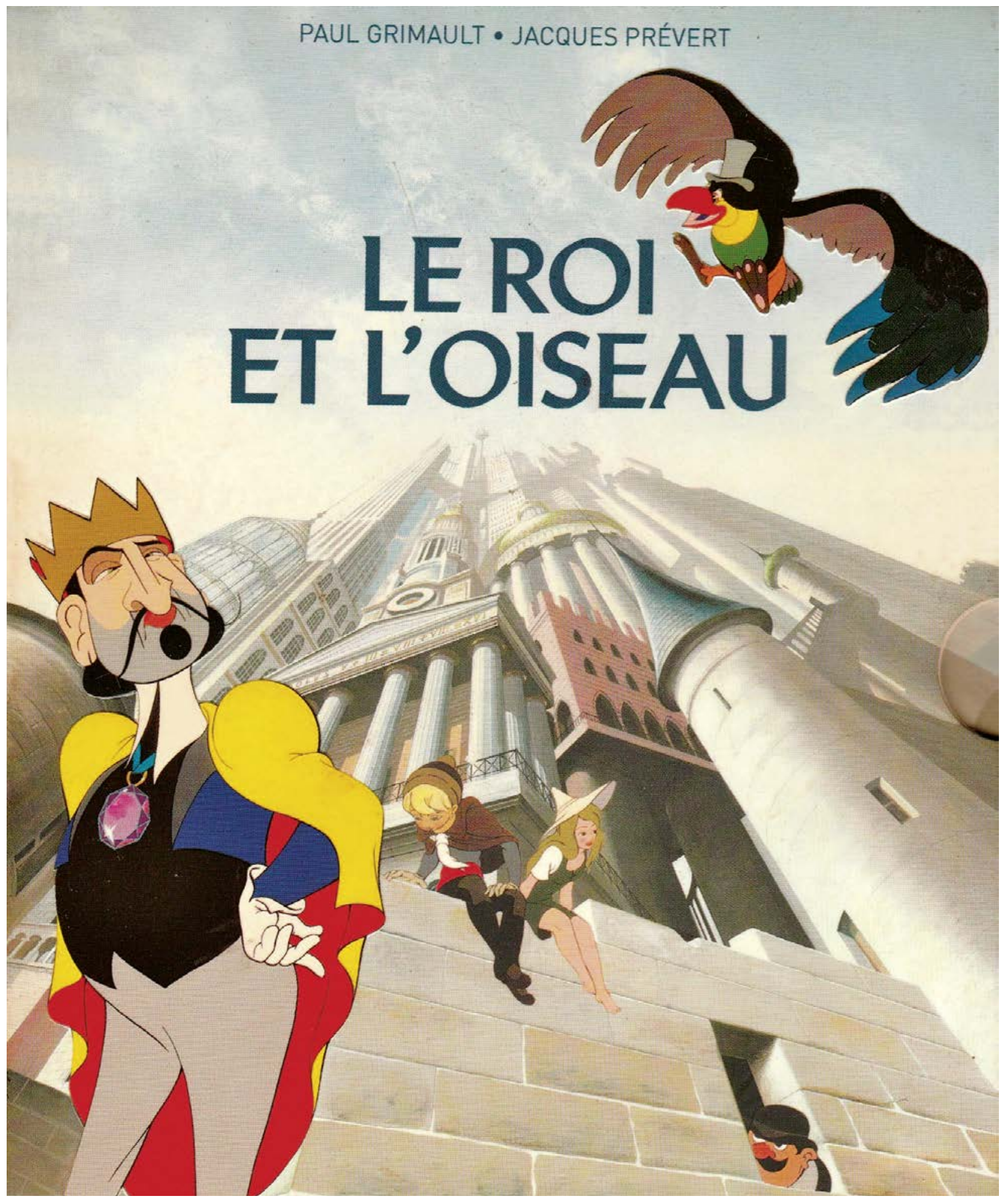




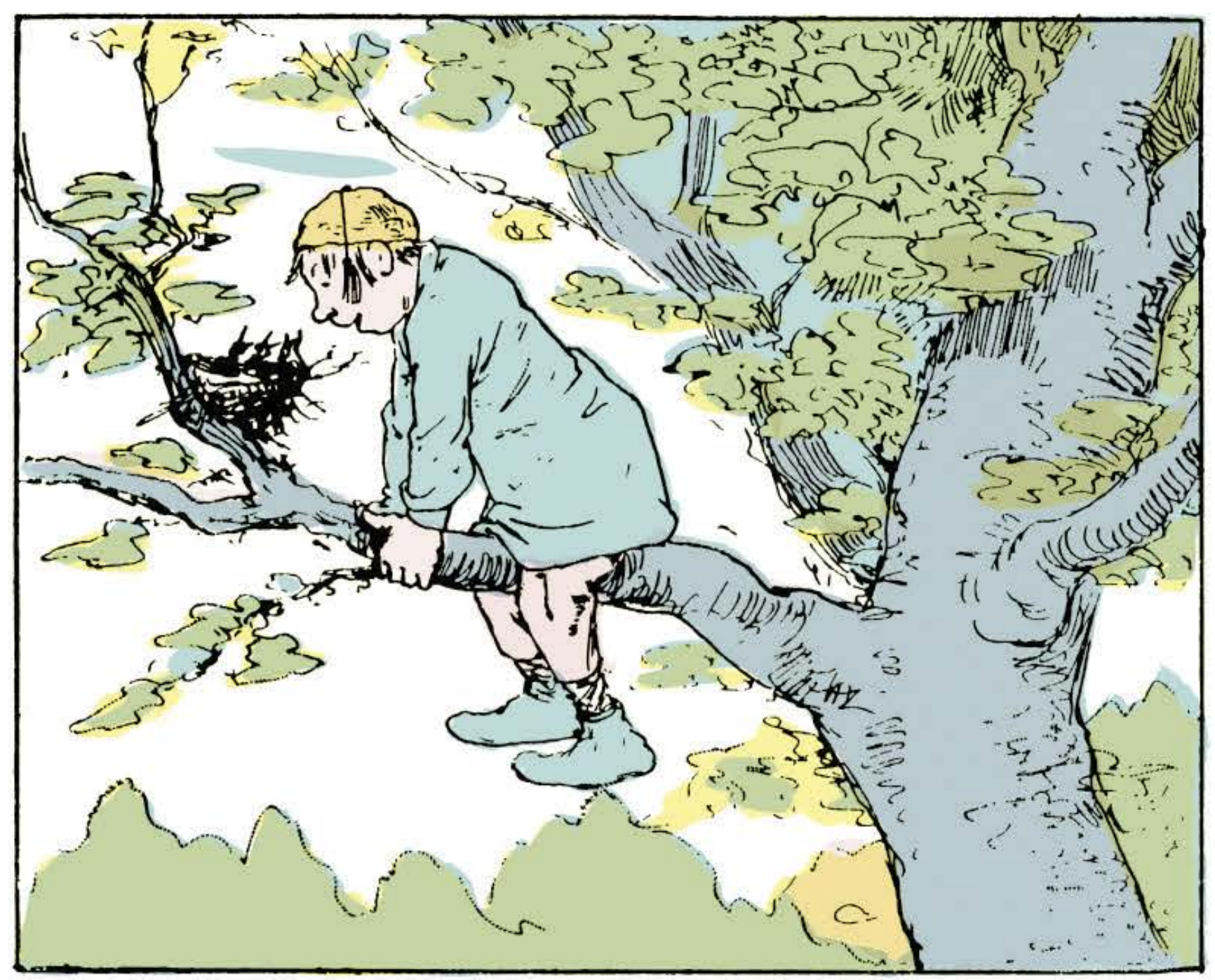

fig. 6

Christophe, "Le Dénicheur

d'oiseaux" in Les Facéties

du sapeur Camember

(c) Armand Colin, Paris,

1986. 
l'analyste des formes très concrètes de la survie en écriture ${ }^{\mathbf{2 3}}$, échafauder un vaste programme européen d'enquêtes sur la diversité des façons contemporaines d'éprouver la «présence» de l'écrivain - et sa «sacralité» - et de restituer ce qu'il appelait l'«aura perdue» de la littérature. Puis, toujours animé du souci de transmettre en les élucidant des expériences fondatrices à travers rencontres, rééditions, éditions critiques, expositions, il revisitera avec Jean Guilaine, Jean-Pierre Piniès, Christiane Amiel - en somme le «groupe de Carcassonne» - cette «poésie des carrefours» (expression empruntée à Michel Leiris ${ }^{24}$ ) qui a infléchi son propre style de chercheur. Notons, parmi tant d'autres, la belle exposition qui réanimait, en 2011, à la Maison des mémoires, l'art de la conversation du poète René Nelli et les journées d'études qui offrirent l'occasion de déchiffrer, pas à pas, «l'affaire de L'Érotique des troubadours ", à savoir les très vives résistances académiques aux propositions novatrices d'une anthropologie historique de l'amour ${ }^{25}$.

\section{Refonder, rassembler}

Au Centre d'anthropologie de Toulouse qu'il a dirigé jusqu'en 1997, les séminaires de Daniel Fabre ont construit une orientation bien reconnaissable de l'ethnologie de l'Europe qui, sous le nom d'«anthropologie du symbolique", associait un parti pris structuraliste à une très grande attention aux conflits, aux déplacements sociologiques, aux transformations et reconfigurations historiques: la microstoria italienne, à la manière de Carlo Ginzburg, ouvrait la voie à une ethnographie dans les archives tandis que le long Moyen Age de Jacques Le Goff autorisait des mises en relation hardies entre des usages relevant de sociétés fort éloignées dans le temps. L'heure des bilans venue, Daniel Fabre considérait que le principal apport des recherches qui en étaient issues, par rapport notamment à la rupture introduite par Yvonne Verdier, concernait l'analyse de la différence chrétienne. De fait, des pans entiers d'usages, que rien n'identifiait a priori comme religieux, s'avéraient donner une matérialité sensible aux principaux énoncés du christianisme et à ses interprétations dissidentes ${ }^{26}$. Mais Daniel Fabre avait une façon bien à lui d'en déplier savamment toutes les articulations. Il fallait beaucoup d'audace et de science pour lier une coutume carcassonnaise de la jeunesse d'Ancien Régime - la fête du roitelet - à la mythologie chrétienne de l'oiseau témoin de la naissance du Christ, aux lâchers rituels de volatiles dans les églises, en passant par l'éducation des rois et les techniques de chasse, pour retrouver dans les récits d'enfance toutes les étapes du nécessaire passage par la voie des oiseaux et, parallèlement, ses métaphorisations dans la poésie amoureuse des troubadours aussi bien que dans l'univers des livres et des abécédaires. Mais, au terme d'un parcours qui appelait autant de vérifications que d'amplifications, c'est toute la subtilité et la richesse d'une culture déclinée en une diversité d'univers sociaux, à première vue sans commune mesure, qui nous était restituée.

À Paris, dans un second laboratoire qu'il créa en 2001 et dont il prononçait malicieusement l'acronyme Lahic - Laboratoire d'anthropologie et d'histoire de l'institution de la culture -, il ouvrait de nombreux chantiers pour penser l'identification, dans nos mondes contemporains, de ce qui "fait culture», du "corps pathétique de l'écrivain » aux imaginaires que nourrissent les savoirs scientifiques ou, inversement, aux savoirs sur l'homme que produisent les univers de fiction, romans, fables, contes ${ }^{\mathbf{2 7}}$. Repenser, telle la
23. Voir Daniel Fabre, "L'écrivain archivé", Sociétés et représentations 19 ("Lieux d'archive»), 2005: 211-233.

24. Voir Michel Leiris, "Antilles et poésie des carrefours ", in Zébrage, Paris, Gallimard, 1992: 67-87.

25. Journées d'études "René Nelli ou la poésie des carrefours », 21-22 avril 2011 ; Daniel Fabre, "L'affaire de L'Érotique des troubadours. René Nelli anthropologue de l'amour provençal » (à paraître).

26. Voir Giordana Charuty, «Du catholicisme méridional à l'anthropologie des sociétés chrétiennes", in Dionigi Albera, Anton Blok et Christian Bromberger (éd.), L'Anthropologie de la Méditerranée/ Anthropology of the Mediterranean, Paris et Aix-en-Provence, Maisonneuve et LaroseMaison méditerranéenne des sciences de I'homme, 2001: 359-385.
27. Voir Daniel Fabre et Jean-Marie Privat (éd.), Savoirs romantiques. Une naissance de l'ethnologie, Nancy, Presses universitaires de Nancy, 2010; Daniel Fabre et Jean Jamin, «Pleine page. Quelques considérations sur les rapports entre anthropologie et littérature ", L'Homme 203-204, 2012: 579612 ; Daniel Fabre, "Roman régionaliste et région romanesque: frontières de la littérature ", in Sylvie Sagnes (éd.), Littérature régionaliste et ethnologie, Arles, Museon Arlaten-

Ethnopôle Garae-Actes Sud, 2015: 199-218. 
28. Voir Daniel Fabre, "Le dernier des paysans: à propos de l'affaire Dominici ", Lengas, revue de sociolinguistique 61, 2007: 117-135; id., "Chinoiseries des Lumières. Variations sur l'individu-monde", L'Homme 185-186, 2008 : 260-300.
29. En plus de la responsabilité du Lahic qu'il a donc fondé en 2001, Daniel Fabre dirigeait depuis 2013 I'Institut international de l'anthropologie du contemporain (IIAC), grosse unité de recherche associée au Centre national de la recherche scientifique (CNRS) et à l'École des hautes études en sciences sociales (Ehess). Président du Groupe audois de recherche et d'animation ethnographique (Garae), devenu, grâce à une convention signée avec le ministère de la Culture, un ethnopôle et un important centre documentaire installé dans la Maison des mémoires de Carcassonne (Aude), il a également présidé, de 1993 à 1997, le conseil de la Mission du patrimoine ethnologique au ministère de la Culture, puis, de 2004 à 2008, la commission 38 ("Anthropologie et étude comparative des sociétés contemporaines") de l'Institut des sciences humaines et sociales du CNRS. De 2009 à 2012, il a fait partie du conseil d'orientation scientifique du musée du quai Branly.

Outre les activités d'administration de la recherche, Danie Fabre n'a jamais négligé les côtés éditoriaux de celle-ci, membre des comités de rédaction des revues Ethnologie française (de 1976 à 1993) et L'Homme (de 1986 à 1996), et, depuis, 2009, codirecteur de la revue Gradhiva ; avec Claudie Voisenat, il a lancé la collection électronique des Carnets de Bérose, publications sur l'histoire de l'ethnologie éditées par le Lahic avec le concours du ministère de la Culture. chouette de Minerve, l'histoire de l'anthropologie comme connaissance des «mondes finissants ${ }^{\mathbf{2 8}}$ » accompagnait ces changements d'horizon que Daniel Fabre offrait en partage à l'École des hautes études en sciences sociales, au Centre national de la recherche scientifique comme au musée du quai Branly, au ministère de la Culture ${ }^{29}$ ou encore à l'université Tor Vergata de Rome, dans un enseignement dit d'«histoire des religions » où se profilait la figure d'Ernesto De Martino et de sa «fin du monde» (La Fine del Mondo), un des derniers grands travaux auquel se sera voué Daniel Fabre en s'attelant, avec Giordana Charuty et Marcello Massenzio, à sauver une nouvelle fois le projet inachevé de l'anthropologue italien ${ }^{30}$.

La liste des publications collectives qu'il a dirigées est elle-même saisissante. Elles recouvrent les objets que se sont donnés les anthropologues, durant ces trente dernières années, pour penser les grandes transformations des sociétés modernes, qu'il s'agisse de la construction des objets et des méthodes (Vers une ethnologie du présent, Paris, Éditions de la Maison des sciences de l'homme, 1992, avec Gérard Althabe et Gérard Lenclud), des pratiques d'écriture (Écritures ordinaires, Paris, P.O.L, 1993; Par écrit, ethnologie des écritures quotidiennes, Paris, Éditions de la Maison des sciences de l'homme, 1997), des identifications nationales (L'Europe entre cultures et nations, Paris, Éditions de la Maison des sciences de l'homme, 1996; La Fabrique des héros, Paris, Éditions de la Maison des sciences de I'homme, 1998 avec Pierre Centlivres et Françoise Zonabend) ou de notre rapport au passé (Domestiquer l'histoire: une ethnologie des monuments historiques, Paris, Éditions de la Maison des sciences de I'homme, 2000; Une histoire à soi. Figurations du passé et localité, Paris, Éditions de la Maison des sciences de l'homme, 2001, avec Alban Bensa) et aux valeurs patrimoniales ${ }^{\mathbf{3 1}}$ (Les Monuments sont habités, Paris, Éditions de la Maison des sciences de l'homme, 2010, avec Anna luso; Émotions patrimoniales, Paris, Éditions de la Maison des sciences de l'homme, 2013).

Plus encore, Daniel Fabre était un homme de revues. En témoigne, à Carcassonne, l'ethnopôle Garae (Groupe audois de recherche et d'animation ethnographique), ce centre de documentation dont il fut l'un des fondateurs en 1981, situé au deuxième étage de la Maison des mémoires, la grande demeure ancienne des $\mathrm{XVII}^{\mathrm{e}}$ et $\mathrm{XVIII}^{\mathrm{e}}$ siècles du centre ville abritant donc en son sein la chambre du poète surréaliste Joë Bousquet. Une collection unique en France de revues, locales, régionales, nationales et européennes, d'ethnologie de la France et de l'Europe y est mise à la disposition du public, des chercheurs et des étudiants. Dans un texte retraçant I'histoire des revues françaises d'ethnologie, de l'après-guerre à aujourd'hui ${ }^{32}$, Daniel Fabre dévoile quelques-unes des raisons profondes, par rapport au livre souvent «prisonnier de l'exercice universitaire", de son attachement aux principes de la revue et de la forme courte de l'article, et surtout de l'article «neuf» où s'exprime le «pouvoir de découverte» de la démarche anthropologique... La revue, au terme de discussions, de négociations, de réécritures, était pour lui, par excellence, l'atelier collectif d'une élaboration disciplinaire et le lieu décentré où pouvait prendre forme, en toute liberté, une réflexion heuristique aux confins d'autres disciplines. Elle était, au sens propre, un lieu, un objet et un instrument de recherche. 
II mit en pratique cette conviction en publiant un nombre considérable d'articles qui condensent dans une écriture éblouissante des années d'enquêtes, de recherches et de lectures (il fréquentait assidûment les bibliothèques, dont la Bibliothèque nationale de France où, plus qu'ailleurs, il avait sa table de travail) aux multiples tournants. Autour des questions des rites funéraires et d'initiation, des figures de la mort et des revenants, des récits de vie et des sentiers de la création tout comme des messianismes, il fut l'un des maîtres d'œuvre de numéros thématiques qui nous tiennent à cœur : «Le retour des morts» (1987) pour Études rurales; «Apprentissages» (1991) pour Ethnologie française; «Du Far West au Louvre: le musée indien de George Catlin» (2006), «Arts de l'enfance, enfances de l'art» (2009), “Création, fiction $^{33}$ » (2014) pour Gradhiva; “Messianismes et anthropologie entre France et Italie» (2013, avec Marcello Massenzio) pour les Archives de sciences sociales des religions; «Auto-biographie, ethno-biographie» (2010, avec Jean Jamin et Marcello Massenzio) «Connaît-on la chanson? » (2015, avec Jean Jamin) pour L'Homme, sans omettre le volume de cette même revue, «Vérités de la fiction » (2005, dirigé par François Flahault et Nathalie Heinich).

Ces numéros thématiques ne peuvent être réduits à la réunion, autour d'un sujet commun, d'auteurs choisis au pied levé. Chacun lui donnait au contraire l'occasion de conclure, au moins temporairement, une phase d'une recherche initiée bien en amont, dont il avait ciselé le développement, étape après étape, lors de ses séminaires, au Centre d'anthropologie de Toulouse puis de l'École des hautes études en sciences sociales, ne redoutant pas de remettre sur le métier un même argument et de revenir patiemment sur les traces d'une voie déjà, par lui, empruntée, en proposant un angle de vue à chaque fois différent. Son enthousiasme, son immense culture, la rigueur d'une pensée qui se construisait chemin faisant entraînaient à sa suite ses auditeurs et ses collègues dans une réflexion commune.

Les trois numéros de Gradhiva sont nés de ces rencontres et constituent chacun le jalon d'une même recherche aux larges perspectives, qui ouvrent sur d'autres textes, d'autres projets dont la seule évocation le portait ailleurs, vers des terres dont il esquissait déjà le défrichement et que, à jamais, sa disparition aura laissé inachevé.

Le numéro sur la réception en Europe du musée indien du peintre George Catlin sortit au printemps 2006, deux mois avant l'inauguration du musée du quai Branly en juin. Cette parution, dont la date fut choisie en fonction de l'événement qu'elle annonçait, proposait une relecture de ce qui avait eu lieu cent soixante-dix ans plus tôt: l'arrivée à Paris de la «première grande exposition anthropologique visible en Europe», réunissant 585 peintures réalisées par Catlin, portraits d'Indiens et paysages de l'Ouest américain, un millier d'objets et de vrais Indiens lowas donnant à voir et à entendre sur scène leurs danses, leurs chants et leurs paroles. La recherche sur Catlin avait vu le jour dans le cadre d'un séminaire, «L'autre de l'art » (2003-2007), où Daniel Fabre explorait le contenu de la dette contractée par l'ethnologie auprès du romantisme, qui thématisa la figure du dernier, dernier locuteur d'une langue, dernier représentant d'une civilisation en son déclin, «individu-monde». L'intitulé désignait en particulier le processus de constitution de la modernité artistique dont il faisait remonter l'apparition aux artistes
30. La version française de cette édition recomposée paraîtra en 2016 aux Éditions de l'École des hautes études en sciences sociales. Daniel

Fabre replace le projet démartinien au sein d'œuvres majeures pensées comme alternatives à la philosophie marxiste de l'histoire: "Ernesto De Martino, La Fin du monde et l'anthropologie de l'histoire", Archives de sciences sociales des religions 161, 2013 : 147-162.

31. À cela il faut ajouter celles auxquelles, ne ménageant ni son temps ni ses enthousiasmes, toujours attentif aux rapports entre histoire et ethnologie, il a participé, et qui ont fait date: Roger Chartier (éd.), Histoire de la vie privée, t. III, Paris, Seuil, 1986; Pierre Nora (éd.), Les Lieux de mémoire, t. III, vol. II, Paris, Gallimard, 1992; André Burguière (éd.), Histoire de la France, vol. IV, Les Formes de la culture, Paris, Seuil, 1993; Giovanni Levi et Jean-Claude Schmitt (éd.), Histoire des jeunes en Occident, vol. II, Paris, Seuil, 1996.

32. Disponible sur http://www.garae.fr/spip. php?article150.

33. Voir l'À propos que lui consacre Nicolas Adell dans le dernier numéro de L'Homme (217, 2016: 109-122). 
34. Voir Daniel Fabre, "Une enfance de roi ", Ethnologie française 21(4), 1991: 329-414.

35. Id., Bataille à Lascaux. Comment l'art préhistorique apparut aux enfants, Paris, L'Échoppe, 2014; voir également la longue recension que lui consacre Rémi Labrusse dans Gradhiva 22, 2015: 238-240.

\section{ci-contre}

fig. 7

Rosalind Solomon, White Bucket, Johannesburg, 1988 (C) 1988 Rosalind Solomon, www.rosalindsolomon.com. Avec l'aimable autorisation de Bruce Silverstein Gallery, New York. de la seconde génération romantique, George Sand, Gérard de Nerval, Théophile Gautier, Champfleury, Charles Baudelaire, Eugène Delacroix... Reconnaissant dans les œuvres des Indiens lowas ou l'imagerie des assiettes peintes la présence d'une altérité artistique là où personne avant eux n'avait identifié de l'art, ils jetèrent les bases d'un premier primitivisme où prirent progressivement place les productions des «exclus de l'art " primitifs, gens du peuple, fous, enfants... Le choc de la rencontre avec cette esthétique nouvelle leur fit apparaître la nécessité d'interroger les origines de l'art et de décrire le processus créateur. On doit à l'acuité du regard de Daniel Fabre d'avoir identifié, dans leurs pratiques artistiques et leurs écrits, à travers le motif de l'enfant dessinant, l'allégorie de la naissance des formes.

Dans la magnifique synthèse qui introduit le numéro sur l'« enfance de l'art ", Daniel Fabre revient sur le cheminement collectif suivi par ces artistes qui les porta à une réinvention de l'enfance et à l'exploration de ses propriétés singulières - "sensibilité en état d'éveil aigu devant le monde", correspondance des sensations, expériences synesthésiques -, en lesquelles ils reconnaissaient une même "langue natale", celle qui habite tout créateur. Convoquant préhistorien, historien de l'art et de la culture, psychologue ayant rencontré l'enfance aux détours de leurs travaux au tournant $d u x x^{\circ}$ siècle, il montrait comment cette altérité qu'«incarne l'enfant auprès de nous et en chacun de nous" fut au cœur des questions anthropologiques sur les commencements de l'art.

L'horizon de l'enfance, ses émerveillements et ses apprentissages, traverse son œuvre. Remontons de nouveau son cours jusqu'à cette «invisible initiation " qui prenait forme dans l'exploration du monde des oiseaux et dont l'évocation revient dans nombre de ses écrits. Ainsi, au numéro d'Ethnologie française (1991), "Apprentissages ", où il avait rassemblé un ensemble d'articles en hommage à Yvonne Verdier, il a donné sa remarquable lecture du Journal de Jean Héroard, médecin, puériculteur et pédiatre de l'enfant roi et futur Louis XIII. «Une enfance de roi» révélait à ses lecteurs ce texte étonnant, où la fascination précoce du dauphin pour les oiseaux témoignait de la prégnance de cette voie initiatique jusque dans les mœurs des cours royales. Héroard, avec une conscience extrême, nota scrupuleusement, dès la naissance de l'enfant, ses faits, gestes et paroles et, précédant de trois siècles les premières analyses des psychologues de l'enfance, en consigna les dessins ${ }^{34}$.

Dans son dernier livre, marchant sur les pas de Georges Bataille à Lascaux ${ }^{35}$, Daniel Fabre poursuit sa réflexion sur l'enfance et l'«autre de l'art ": il y interroge la quête, par l'écrivain et philosophe, de l'émotion produite au moment originel de la découverte, les conditions de visibilité de ces grottes aux murs ornés, contemporaines d'autres apparitions, mais qui par une «étrange coïncidence» font que ces peintures enfouies, enterrées, cachées pendant des millénaires se sont éveillées, à Altamira, à Lascaux, sous le regard et à la suite de gestes intrépides d'enfants. Comme si la préhistoire devait sauter aux yeux de petits d'hommes courant après leur histoire à venir, après ce qui deviendra leur âge d'homme: une allégorie magnifique de l'initiation, entre ce qui s'expose et se masque, entre ce que l'on quitte et ce qu'on acquiert, entre puérilité et maturité. 


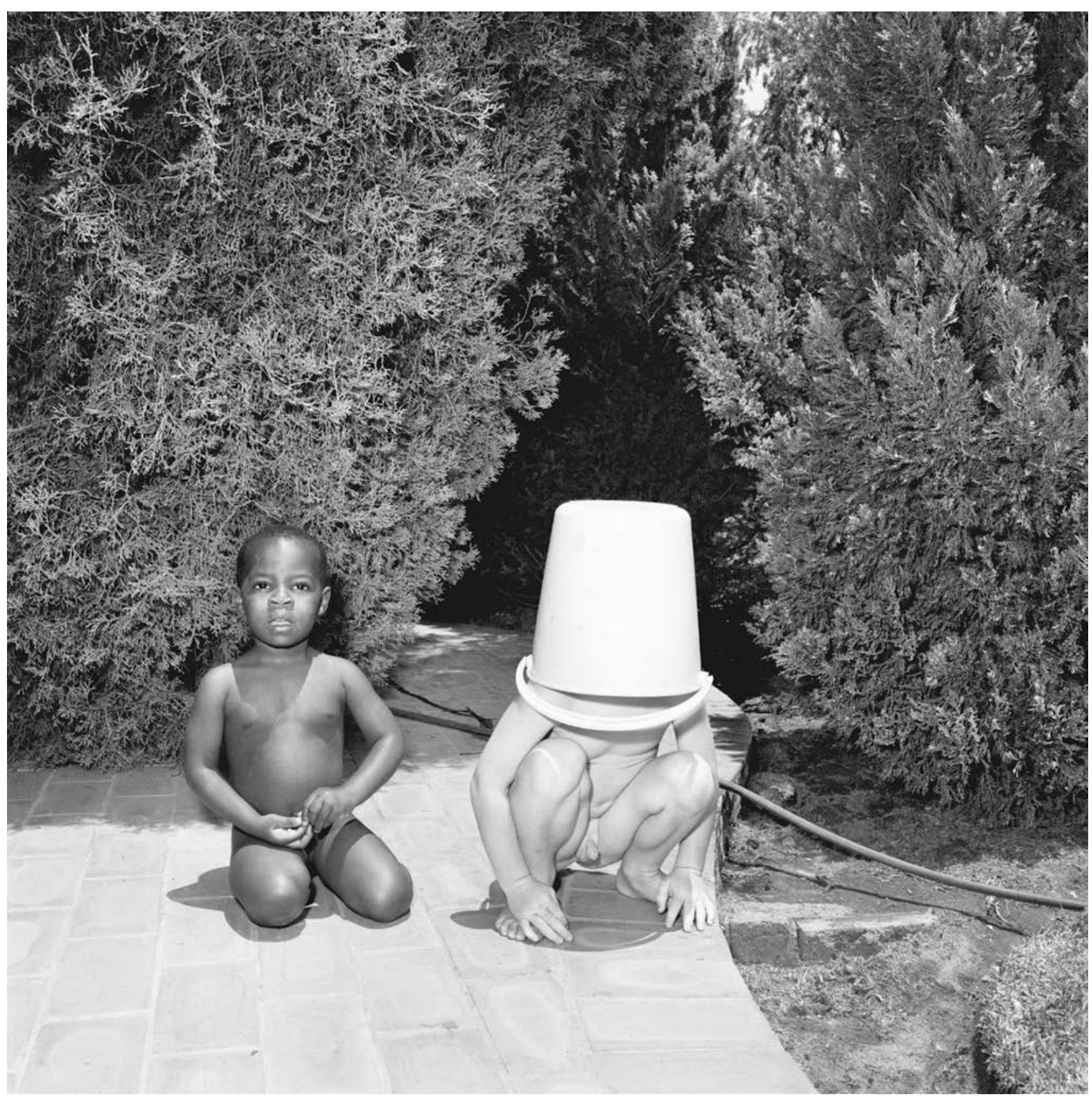




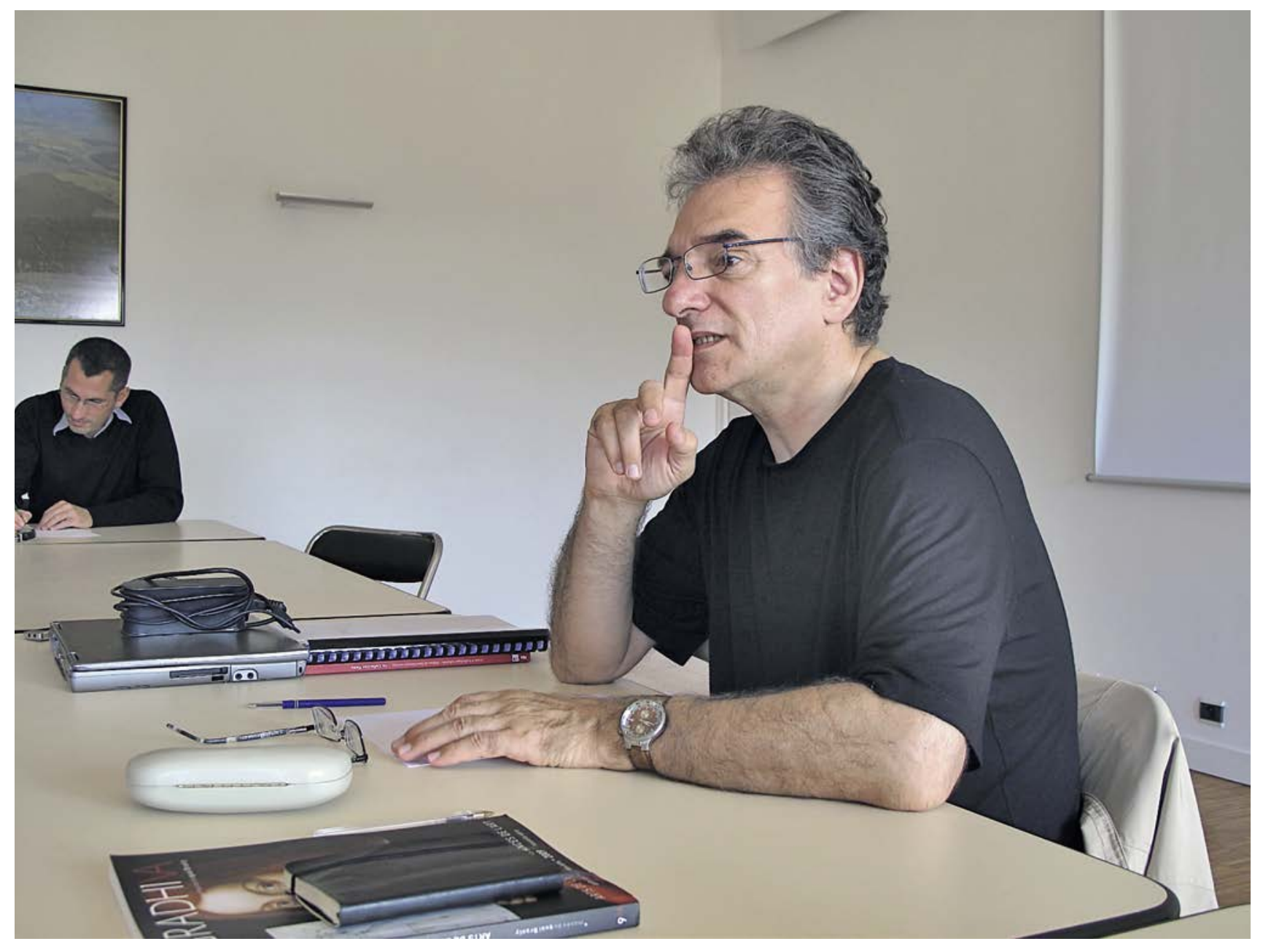


Suivant le fil rouge de la relation de l'artiste à ses propres commencements, celui des conditions du surgissement créatif et des fictions élaborées pour les dire, Daniel Fabre revient dans Gradhiva, "Création, fiction "(2014), sur l'expérience physique d'écrire et l'évidence du corps dans la «traversée créatrice ${ }^{\mathbf{3 6}}$ ». Jean-Jacques Rousseau, François-René de Chateaubriand, Honoré de Balzac ou Gustave Flaubert font alors place à Marcel Proust «en mal de mère", assisté, et ethnographié, par sa servante Céleste Albaret dans son travail de création, qui est celui d'une parturition. «Le travail nous rend un peu mère", écrivait Proust en 1908. Comment ne pas percevoir dans ce retour sur la construction et les souffrances du sujet créateur une de ces traces autobiographiques évoquées précédemment? Daniel Fabre avait cependant choisi le détour par la fiction narrative pour approcher la vérité de l'acte créatif. II avait promis d'autres développements à cette anthropologie des arts, de la création et de la littérature à l'enrichissement de laquelle ses travaux ont apporté une contribution majeure: le traitement par la littérature - Jean Giono, Ferdinando Camon (ce dernier déjà présent en 2010 dans le numéro de L'Homme, "Auto-biographie, ethno-biographie") de la figure de l'artiste populaire aux prises avec sa propre création.

\section{L'invisible initiation}

Daniel Fabre était aussi un admirable conférencier. Le 9 février 2015, pour le public du campus Condorcet, il recomposait, de manière lumineuse, une recherche touffue qui dialoguait avec l'œuvre d'Yvonne Verdier, l'anthropologie historique médiévale et les historiens de l'Ancien Régime pour identifier les apprentissages juvéniles dans nos sociétés dépourvues, croyait-on, de rites d'initiation. À cette négation, il oppose fermement le «trajet initiatique discret et même invisible " qui, dans les sociétés rurales européennes, permettait aux garçons d'effectuer le passage à l'âge d'homme: «des expériences de transgression des frontières - entre sauvage et domestique, vivants et morts, masculin et féminin ", que le carnaval rend plus ostensibles, mais qui ne s'y réduisent pas.

Si l'on peut mettre en évidence une «loi du silence» dans des sociétés à "grande initiation", notamment africaines (la problématique du secret), Daniel Fabre a décelé, pour sa part, une «loi d'invisibilité » (la problématique de l'écran) dans les sociétés à initiation "diffuse» (comme celle qui a cours dans nos propres société et culture) - celle-ci, pendant ou corollaire de celle-là (registre de la parole pour l'une, registre de l'image et de l'écrit pour l'autre), mais qui, contrairement à l'opinion commune ou à un fonctionnalisme béat, placent toutes deux le processus initiatique sous le signe de la négativité, ne serait-ce que par le fait que, de façon générale, toute initiation agit moins comme un rite de passage que comme un passage par le rite... Ce n'est pas de dire qu'on est ceci (mettons adulte) qu'il s'agit, mais d'affirmer qu'on n'est plus cela (disons «un petit», un enfant): l'âge d'homme ne s'acquiert, ne se conquiert qu'a posteriori, comme l'avait magistralement démontré l'ethnographe et écrivain Michel Leiris, qu'admirait Daniel Fabre ${ }^{\mathbf{3 7}}$. Ce qui, dans la constitution même de l'individualité et de l'identité, introduit une temporalité mémorielle. D'où la recherche qu'il avait engagée sur cet autre phénomène oral, écrit, ou pictural même, que représentent la biographie et l'autobiographie, et sur leur performativité sociale, voir socialisante. À ce titre, ses réflexions sur l'oralité, la narrativité, la littéralité, la monumentalité,
36. Voir Daniel Fabre, "Le corps pathétique de l'écrivain ", Gradhiva 5, 1999: 1-13. Id., "L'androgyne fécond et les quatre conversions de l'écrivain ", in Clio. Femmes, genre, histoire 11 ("Parler, chanter, lire, écrire »), 2000, disponible sur: http://clio.revues. org/214 ; DOI : 10.4000/ clio.214.

37. Au moment où la mort l'a surpris, il était en train d'écrire, pour Gradhiva, une longue recension de l'exposition Leiris \& Co: Picasso, Masson, Miró, Giacometti, Lam, Bacon... du Centre Pompidou-Metz qu'il avait longuement visitée le 12 avril 2015, et de l'important catalogue qui l'accompagnait (Paris, Gallimard, 2015).

\section{ci-contre}

fig. 8

Daniel Fabre lors des journées d'études du Lahic à Bibracte (Morvan), septembre 2009 (cl. Jean Jamin). 
38. Voir Jacques Réda, Battement, SaintClément-de-Rivière, Fata Morgana, 2009.

39. Voir Michel Leiris, "Le sacré dans la vie quotidienne " et "L'homme sans honneur ", La Règle du jeu, Paris, Gallimard, 2003 (édition de Denis Hollier) : 1110-1118 et 1119-1154.

40. Voir Robert Hertz, Mélanges de sociologie religieuse et de folklore, Paris, Félix Alcan, 1928.
41. Voir également la scène $V$ de l'acte IV de Hamlet de Shakespeare, où la «blanche Ophélie » chante sa détresse. sa mélancolie, sa nostalgie avant de mourir noyée au milieu d'une guirlande de fleurs et de rameaux qu'elle avait cueillis puis tressés comme une couronne, et dans les plis de sa longue robe que les flots venaient alourdir: "On dit que la chouette a été jadis fille d'un boulanger ", murmuret-elle, se souvenant d'une légende paysanne du Gloucestershire. "Seigneur, ajoute-t-elle, nous savons ce que nous sommes, mais nous ne savons pas ce que nous pouvons être. "

42. Voir Daniel Fabre et Jean Jamin, "Chanter soir et matin... ", L'Homme 215-216, 2015: 7-14; Daniel Fabre, "Que reste-t-il ? Quatre figures de la nostalgie chantée", ibid. : 15-46; id., "Rock des villes et rock des champs ", ibid. : 233-250.

\section{ci-contre}

fig. 9

Les Héros de la pensée, un projet de Massimo Furlan et de Claire de Ribaupierre. Centre d'art de Neuchâtel, janvier 2012, avec Marc Augé, Pierre-Olivier Dittmar, Daniel Fabre, Bastien Gallet, Emmanuel Giraud Jacques Heinard, Serge Margel, David Zerbib

(c) Photo Sully Balmassière. la patrimonialité et la sacralité nous semblent renouveler totalement notre conception et notre anthropologie des mondes contemporains, en mettant l'accent sur leur composition sociale (au sens musical du terme), c'est-à-dire sur leurs lignes mélodiques (historicité) autant que sur leurs grilles harmoniques (transhistoricité), sans oublier leurs rythmes (circularité) et battements (aspérités: entre rectitude et cassure ${ }^{\mathbf{3 8}}$ ). En ce sens, Daniel Fabre a parfaitement prolongé et ressourcé le programme que, sur un plan essentiellement autobiographique, littéraire et poétique, le même Leiris s'était quant à lui fixé, le premier questionnant les transferts du sacré non seulement dans la vie quotidienne comme le fit Leiris ${ }^{39}$, mais dans la vie sociale, culturelle, en l'occurrence artistique - ce que Robert Hertz ${ }^{40}$ a appelé le sacré gauche.

Autre grand et dernier chantier qu'avait ouvert récemment Daniel Fabre, à la fois dans un séminaire de l'École des hautes études en sciences sociales et dans un numéro de L'Homme $(215-216,2015)$ où figurent ses toutes dernières publications, celui de la chanson populaire telle qu'elle s'est déployée à l'âge de la musique enregistrée. Des perspectives générales sur ce «genre" spontanément identifié comme tel étaient proposées par lui, en soulignant en particulier la tension entre l'illégitimité culturelle, voire épistémologique, de la "chanson de variétés" (en italien musica leggera) et sa place dans la construction des mémoires et des identités. "Air du temps", "bruit de fond de l'histoire", "yo-yo de la mémoire", "sémaphore de la sensibilité", la chanson tient une place centrale dans l'économie des émotions auxquelles elle finit par conférer un langage qui, comme tout langage, articule une face sociale - communicationnelle et communielle - et une face intime, tournée vers l'expression et l'élucidation de la singularité du sujet, dans l'acception moderne du terme. Michel Leiris ne disait-il pas que chanter est le plus court chemin pour aller de soi à soi? Dans cette perspective, Daniel Fabre a proposé une fine analyse des rapports entre chanson et nostalgie, en rappelant que l'épreuve psychologique de la nostalgie est dès l'origine - à la fin du xVIII' siècle - liée à l'écoute musicale de chansons du pays natal par des exilés. Dans ce contexte, la chanson a tous les traits contradictoires et complémentaires du pharmakon, cause et remède du mal. La démonstration en est faite par Jean-Jacques Rousseau dans son œuvre autobiographique et musicologique. Partant de la thèse rousseauiste - «l'usage des chansons semble être une suite naturelle de celui de la parole" -, l'analyse porte sur le rôle des premières chansons apprises dans l'enfance et l'adolescence, insistant sur l'embarras qui saisit le sujet qui n'arrive pas à en retrouver l'air puis les paroles. Situation étrange et douloureuse où l'on «a la mémoire de ce dont on ne se souvient pas " (Pascal Quignard) et qui fait de ces chansons des équivalents du «nom propre», désignatif d'un objet unique et chargé de connotations singulières, mais dont personne, pas même soi, ne connaîtra jamais le sens: "Je m'appelle» deviendrait une sorte de "Je me chante», ou plutôt, à la manière de Rimbaud: "On me chante 41 "! Daniel Fabre montre comment cette expérience fondatrice $a$ fait du thème de la nostalgie le cœur même de la chanson contemporaine et de ses usages dominants ${ }^{42}$.

\section{As time goes by}

Son approche dynamique des phénomènes de socialisation et de construction de l'identité, qu'elle soit sociale, ethnique, sexuelle ou religieuse dans des mondes locaux en prise directe avec ce qu'on appelle la globalisation, 

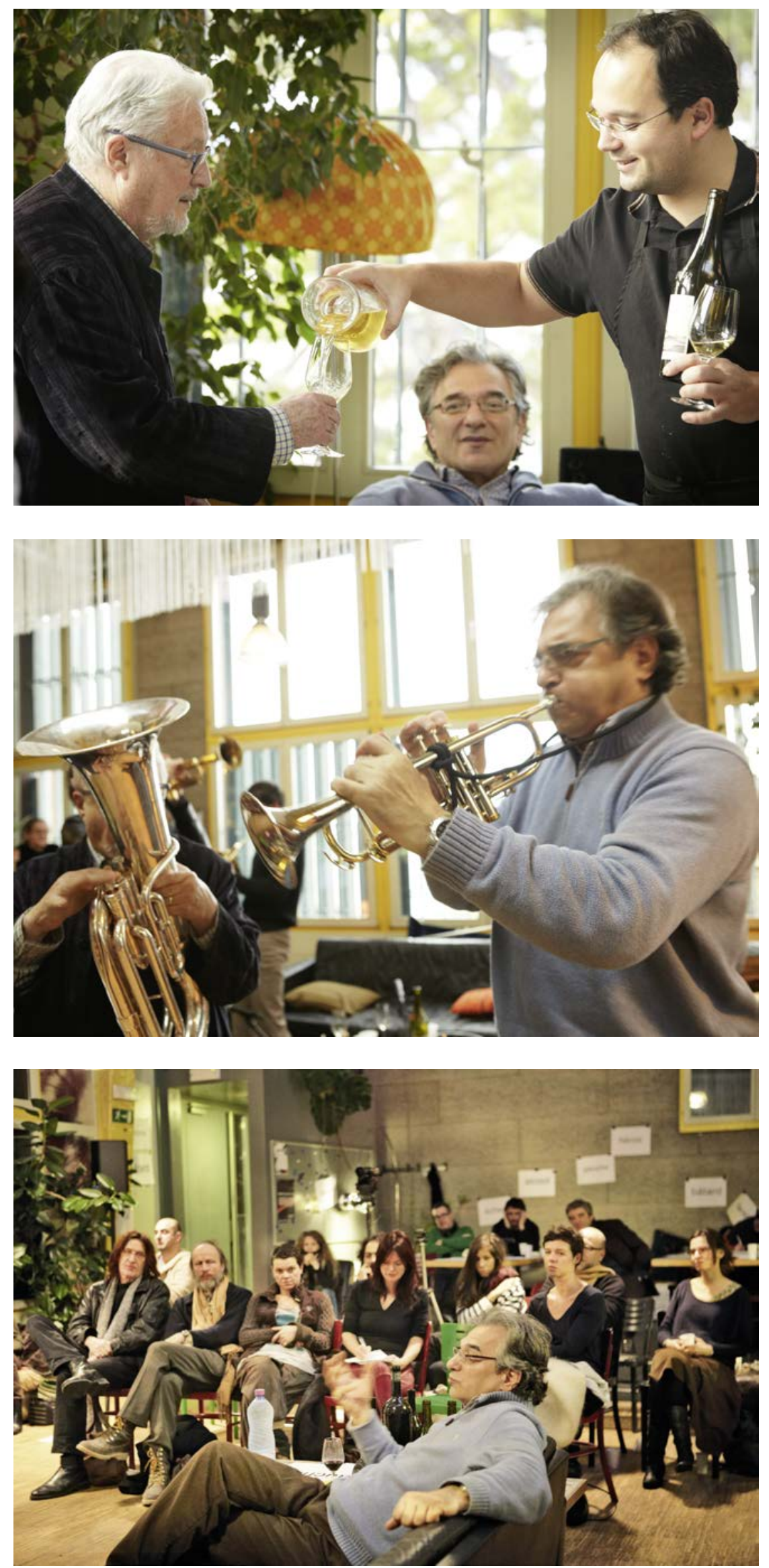


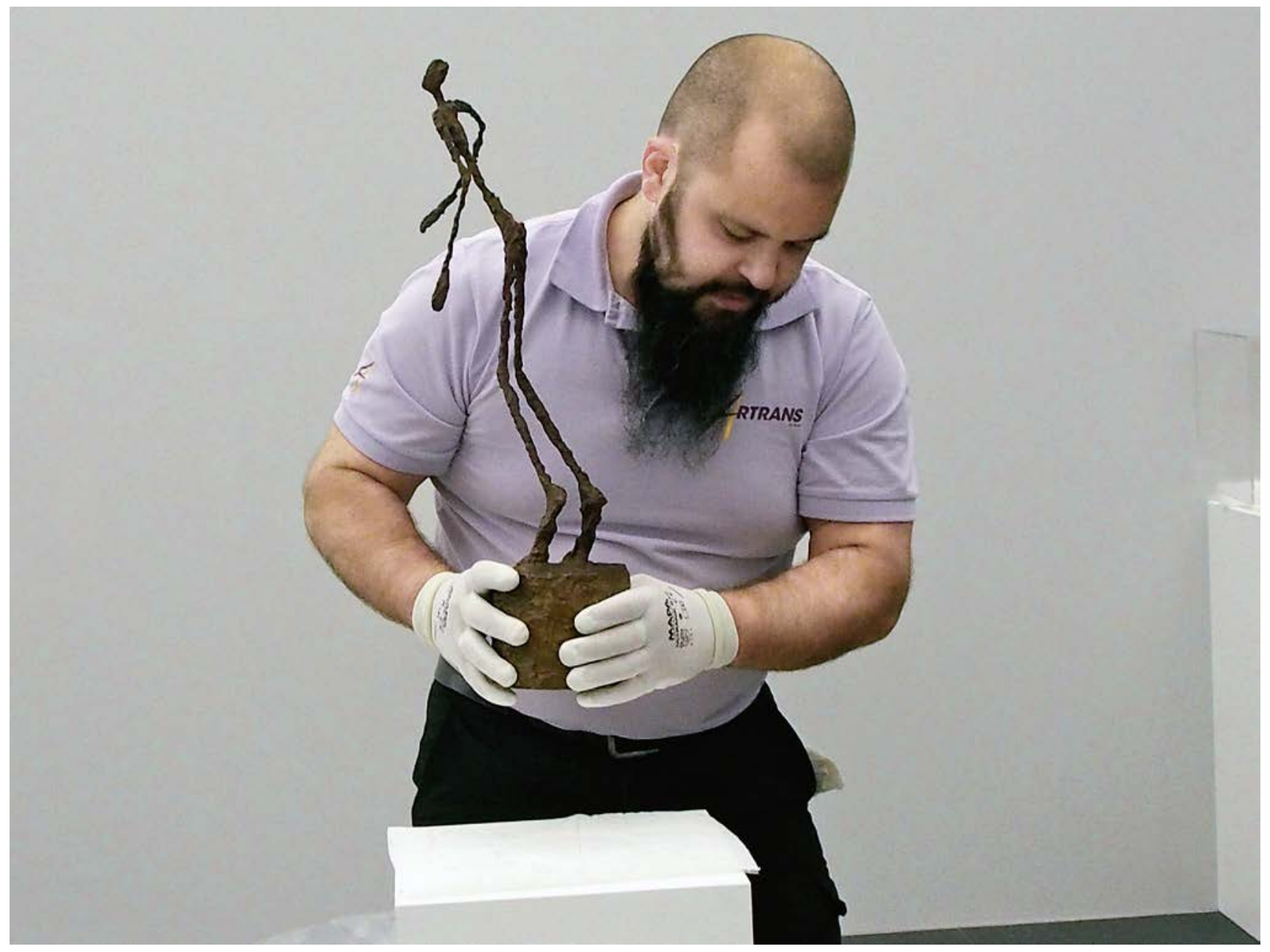

fig. 10

Installation de L'Homme qui

chavire, bronze d'Alberto

Giacometti (1950),

à l'exposition Leiris \& Co,

Centre Pompidou-Metz,

avril 2015 (cl. Jean Jamin)

(C) Succession Alberto

Giacometti (Fondation

Alberto et Annette

Giacometti, Paris)

ADAGP, Paris 2016. 
ses analyses toujours circonstanciées, rigoureusement documentées, pertinemment contextualisées de leurs transformations, recompositions ou cristallisations rituelles et artefactuelles, ont permis à Daniel Fabre - empruntant les chemins défrichés par Norbert Elias ou Ernesto De Martino dont il avait analysé la difficile réception en France ${ }^{\mathbf{4 3}}$, sans cependant s'y contenir - de repenser les questions de la tradition et de la modernité, voire de la postmodernité: notions «fortes» et focales de l'anthropologie, mais qui, pour être pertinentes et heuristiques, exigent une mise à l'épreuve descriptive et comparative. Ce qu'il a su opérer à partir de ses nombreuses et riches expériences de terrain. Et ses fulgurances, méthodiquement référencées, vérifiées, pouvaient ouvrir, pour chacun, ce qu'il appelait tout récemment «le chemin du plaisir de la vie» où les expériences - existentielle, artistique et intellectuelle - étaient réconciliées ${ }^{44}$.

Son éclipse (hélas définitive) nous incite à nous remettre vite au travail et, surtout, à le relire autour d'un verre ou deux de madiran (il aimait ça!) plutôt qu'avec un crayon dans une main et la tête penchée sur l'autre, comme on écoute ou lit un faiseur de contes. Daniel Fabre n'avait pas son pareil pour nous raconter l'histoire, les histoires de l'ethnologie qu'il faisait défiler comme on effeuille un recueil de fabliaux, mais avec une hauteur de vue et une mise en perspective rares, qui les soustrayaient à la morosité et à la monotonie archivistiques.

Homme de science certes, Daniel était aussi homme d'amitié, de proximité, de convivialité: ses séminaires avaient la chaleur des veillées qu'il enrichissait d'ann...nées en ann...nées de son accent occitan. Monsieur Fabre sera toujours là.

EPHE - LAHIC - IIAC giordana.charuty@laposte.net

CNRS - LAHIC - IAC michele.coquet@cnrs.fr

EHESS - LAHIC - ॥AC jean.jamin@orange.fr
43. Voir Daniel Fabre, "Un rendez-vous manqué. Ernesto De Martino et sa réception en France ", L'Homme 151, 1999: 207-236.

44. Avec, entre autres, Marc Augé et Jacques Hainard, il avait participé au spectacle-performance conçu par Massimo Furlan et Claire de Ribaupierre, créé au Centre d'art de Neuchâtel en janvier 2012, puis joué au théâtre de la Cité internationale de Paris en octobre 2012, et intitulé Les Héros de la pensée. Utilisant la forme de l'abécédaire, en hommage à Gilles Deleuze, ce spectacleperformance consistait à tirer au sort un carton parmi les vingtsix présentés au public, sur chacun desquels était écrit un concept ou une idée, voire un simple mot commençant par une des vingt-six lettres de l'alphabet, et de demander aux huit acteurs d'improviser tour à tour pendant une heure sur le terme en question, après quoi les participants étaient invités à manger et à boire un verre de vin (ou deux) de vingt-six appellations et robes différentes, et ce pendant plus de trente heures d'affilée: la résistance à la fatigue, à l'alcool, à la nourriture, au sommeil, aux pressions du public était une manière pour les «performateurs" de se mettre en posture héroïque et d'aller. si l'on peut dire, jusqu'au bout de leur pensée (à Neuchâtel ils devaient en outre souffler dans un cuivre - tuba, euphonium, trompette, trombone, cor - entre chaque tirage au sort pour produire des sons qui, certes, n'avaient rien d'harmonieux puisque aucun des huit participants-acteurs ne pratiquait l'instrument). Daniel Fabre fut dans tous ces exercices un des plus «performants». 